\section{Cureus}

Review began 10/16/2012 Published 11/26/2012

\section{C) Copyright 2012}

Hathout et al. This is an open access article distributed under the terms of the Creative Commons Attribution License CC-BY 3.0., which permits unrestricted use, distribution, and reproduction in any medium, provided the original author and source are credited.

\title{
Transient Tumor Volume Increase in Vestibular Schwannomas after Radiotherapy
}

Lara Hathout ${ }^{1}$, Carole Lambert ${ }^{2}$, Jean-Francois Carrier ${ }^{3}$, Jean-Paul Bahary ${ }^{3}$, Yannick Hervieux ${ }^{3}$, Robert A. Moumdjian ${ }^{4}$, Marie-Andree Fortin ${ }^{3}$, David Roberge ${ }^{5}$

1. Department of Radiation Therapy, CHUM - Hôpital Notre-Dame, Centre hospitalier de l'Université de Montréal (CHUM) 2. Department of Radiation Oncology, Centre hospitalier de l'université de Montréal (CHUM) - Hôpital Notre-Dame 3. Department of Radiation Oncology, Centre hospitalier de l'Université de Montréal (CHUM) 4. Department of Neurosurgery, CHUM - Hôpital Notre-Dame, Centre hospitalier de l'Université de Montréal (CHUM) 5. Department of Oncology, Division of Radiation Oncology, McGill University Health Center

$\square$ Corresponding author: Lara Hathout, lara.hathout@gmail.com Disclosures can be found in Additional Information at the end of the article

\section{Abstract}

Background: Transient tumor progression is difficult to diagnose and is challenging for the physician.

Objectives: To report and review the transient tumor progression rate and induced toxicities in patients treated for vestibular schwannomas (VS).

Materials and Methods: From January 2005 to February 2010, 43 patients with VS were consecutively treated at our institution. Twenty patients (46.5\%) were treated with fractionated stereotactic radiation therapy (FSRT) using a LINAC or a CyberKnife technique with doses varying between 18 and 50.4 Gy. Radiosurgery (SRS) on a conventional LINAC and on a CyberKnife were performed in 13 patients (30\%) (median dose of $12 \mathrm{~Gy}$ ). Tumor volume increase was calculated from the first tumor volume increase until tumor stability or regression. A Fisher's exact test and Mann-Whitney U test were used for statistical analysis.

Results: Transient tumor volume increase occurred in 10 patients (25\%) with a median time to tumor enlargement of 5.5 months (1-25 months). Prior surgical resection was significantly associated with transient tumor volume increase (Fisher exact, $\mathrm{p}=0.02$ ). Increased rates of facial and trigeminal neuropathies were associated with transient tumor volume increase $(\mathrm{p}=0.04$ and $\mathrm{p}=0.08$, respectively). The pseudoprogression rate and the cranial nerve toxicity were not associated with any dosimetric factor.

Conclusions: Transient tumor volume increase is frequent (25\%) after radiotherapy and significantly associated with previous resection possibly due to the surgical microvascular insult. Cranial nerve toxicities are significantly correlated with, and possibly caused by, transient tumor progression.

Categories: Radiation Oncology, Neurosurgery

Keywords: cyberknife, vestibular schwannomas, surgical resection, linac, transient tumor progression 


\section{Introduction}

Vestibular schwannomas (VS) are benign intracranial tumors, which represent $8 \%$ of adult intracranial tumors. With a growth rate approaching $2 \mathrm{~mm}$ per year [1], tumor progression can lead to progressive hearing loss, tinnitus and vestibular ataxia.

With the exception of large tumors with acute mass effect, management options can lead to controversy. Surgical resection is often proposed to medically fit patients [1] for whom it has a low mortality rate $(0.3-0.6 \%)[2,3]$, a high rate of complete tumor removal and a low probability of recurrence [4-7]. Hearing preservation is achieved in $32-50 \%$ of cases, while permanent facial neuropathy and postoperative CSF leak occur in $14-29 \%$ and $6-11 \%$, respectively $[2,3,8,9]$.

Conservative management with serial MRIs is valid option in older patients with a short life expectancy, slow growing, small to medium-sized tumors, and a low symptomatic burden. Whether of not delayed treatment results in worse ultimate cranial nerve function is debatable. In observed patients, tumor growth will lead to further permanent hearing loss [10] in 29-54\% of patients. Sixteen to twenty-six percent of observed patients will eventually require tumordirected treatment $[2,11-15]$.

New studies with Bevacizumab reported promising results in patients with type 2 Neurofibromatosis and evidence of progressive VSs that were considered poor candidates to surgery and radiation therapy [16].

Stereotactic radiosurgery (SRS) is a common alternative to surgical resection. It can be delivered using an isocentric linear accelerator (LINAC), a GammaKnife or a CyberKnife robotic radiosurgery device (CK). Patients with small $(<2.5 \mathrm{~cm})$ tumors without significant brainstem compression are eligible for stereotactic radiosurgery (12-13 Gy).

Fractionated stereotactic radiotherapy (FSRT) is a safe alternative for selected patients illsuited to radiosurgery because of tumor size or mild brainstem compression, Controversy exists as to the relationship of fractionation and hearing preservation - most departments where FSRT is available will recommend fractionation to patients with serviceable hearing. Dose schedules vary considerably - 18-54 Gy in three to 30 fractions. Independent of the selected schedule, local control is expected in 90-98\% [17-21]. The main advantage of CK over other radiation techniques is that there is no technical compromise when delivering a fractionated course of radiation.

Transient tumor progression following radiation is commonly reported, although the underlying physiopathology is poorly acknowledged. Such transient progression, which can last up to two years, has been well-described after single fraction SRS. There is limited data concerning transient progression following FSRT.

The objective of our study is first to report our transient tumor progression rate as well as the contributing factors in patients treated with stereotactic radiotherapy. Second, we will review the correlation between transient tumor progression and the adverse reactions observed after radiation.

\section{Materials And Methods Characteristics of the patients}

From January 2005 to February 2010, 43 patients with VS were consecutively treated with FSRT or SRS at our institution with a minimum follow up of two years. Radiation was used as primary treatment in 23 patients (53.5\%), as salvage treatment following surgery in nine patients (21\%) 


\section{Cureus}

and at progression following observation in 11 patients (25.5\%). Five patients with neurofibromatosis type 2 were included in our study. Median age at diagnosis was 56 years. Prior to RT, 16 patients (37.2\%) had serviceable hearing - Gardner-Robertson Grade 1-2. Patient and tumor characteristics are detailed in Table 1.

\section{Characteristics}

Neurofibromatosis type 2

Tumor localisation

Right

Left

$16(37 \%)$

Cranial nerve neuropathy at diagnosis

CN V

CN VII

CN VIII

CN IX, X and XII

Tumor size

$<2 \mathrm{~cm}$

$2-4 \mathrm{~cm}$

$>4 \mathrm{~cm}$

Radiation therapy fractionation

12-30 fraction FSRT

3 fraction FSRT

SRS
$8(19 \%)$

$18(42 \%)$

$8(19 \%)$

$5(12 \%)$

$42(98 \%)$

$11(26 \%)$

$2(5 \%)$

$10(23 \%)$

$15(35 \%)$

TABLE 1: Patients and treatments characteristics

Surgical resection prior to radiotherapy had been performed in 9 patients (20.9\%) for a total of 12 surgeries ( 3 patients had 2 surgeries prior to RT) of whom 3 had gross total resection and 9 had subtotal resection.

Tumor size was measured according to the cerebellopontine angle maximum method (CPA). The tumor including the extracanalicular component was measured in the maximum anteroposterior and medial-lateral diameters on axial MRI. . The majority of patients (28 patients, 65\%) had maximum tumor diameter $£ 20 \mathrm{~mm}, 11$ (25.5\%) patients had tumors 20-40 $\mathrm{mm}$ and 2 (4.6\%) had tumors $>40 \mathrm{~mm}$. 
At diagnosis, 8 (18.6\%) and 5 (11.6\%) patients had a trigeminal or facial neuropathy, respectively. Toxicity was assessed and graded according to the Common Terminology Criteria for Adverse Events (CTCAE v3). Facial nerve palsy was graded according to the HouseBrackmann score.

\section{Radiotherapy techniques}

All patients had a contrast-enhanced computed tomography (CT) and 3D Gradient Echo Ultrafast MRI with a slice thickness of $1 \mathrm{~mm}$ for treatment planning. Thin slice, contrast CT images in the treatment position were co-registered to the MRI. Later in the series, a True Fast Imaging with Steady state Precession (FISP, CISS or FIESTA) sequence was often co-registered to the $\mathrm{CT}$ and TI contrast imagines.

Twenty patients (46.5\%) were treated with isocentric LINAC-based FSRT using a micro multileaf delivery system (Brainlab, Feldkirchen, Germany). The prescription dose was 45-50.4 Gy in 25-28 fractions for 9 patients and 36 Gy in 12 fractions for 9 patients at the isodose line covering $98-100 \%$ of the PTV. The fractionation regimen of 36 Gy in 12 fractions was used in 2005 and 2006 in our institution as an equivalent biological dose [17] to 45-50 Gy in 25 fractions using an $\mathrm{a} / \mathrm{b}$ of 3 . It was mainly used in older patients and in those who had a transportation limitation.

Radiosurgery using conical collimation on a conventional LINAC (Philips/Elekta) was performed in 7 patients (16.3\%) using an invasive stereotactic localization frame and in 2 patients using a thermoplastic mask. The median dose delivered was $12 \mathrm{~Gy}$ (range 12-14.5 Gy) prescribed to the covering isodose surface and delivered through 3-5 noncoplanar arcs.

FSRT using a Cyberknife technique was performed in ten patients at a dose of $18 \mathrm{~Gy}$ in 3 fractions. Six patients received SRS (12-12.5 Gy) with the Cyberknife. The ultra-short course FSRT (associated with the misnomer "staged radiosurgery") was mainly used in patients with serviceable hearing and/or larger tumors in contact with the brainstem.

For each treatment approach, the dose, fractionation, prescription isodose line, maximal dose for the PTV, conformity index, homogeneity index, PTV and GTV volume were reported.

\section{Post-treatment clinical assessment}

Most of the patients were seen 6 weeks after their treatment and thereafter at 3 to 6 months interval for the first 2 years and then annually. With the approval of the local ethics review board, all patients were contacted according to a pre-defined telephone protocol. Serial MRIs were requested every 6 months for the first 2 years then once a year or in accordance to clinical findings. Local control was measured from the end of radiation. As described by Flickinger [22], we defined tumor volume increase as tumor growth of $1 \mathrm{~mm}$ in any two directions or $2 \mathrm{~mm}$ in one direction. Tumor enlargement due to treatment failure usually occurs several months after radiation and tends to persist for a greater period than two years whereas transient tumor volume increase occurs in a relatively acute setting and stabilizes within two years. Therefore, local control was calculated using the last follow up MRI and transient tumor volume increase was calculated from the first tumor volume increase until stability or regression of the tumor. Patients who had systematic tumor volume increase without stability or regression were considered to have treatment failure. Tumor stability was defined as no change in tumor size on serial MRIs.

\section{Statistical analysis}

The Kaplan Meier method was used to estimate local control. A Fisher's exact test was 


\section{Cureus}

\section{Factors evaluated}

Prescription dose

GTV volume

Maximum dose to PTV

BED

Age at treatment

NF 2

Gender

Previous surgery

Facial nerve toxicity

Trigeminal nerve toxicity performed to validate statistical correlation and Mann-Whitney U test was performed to compare the different treatment technique. All tests were two-tailed. SPSS 17.0 was used (IBM, Armonk, New York)

\section{Results}

\section{Transient tumor volume increase}

In order to estimate the transient tumor volume increase rate, we excluded the three out of the 43 patients who did not have a control MRI after radiation. Transient tumor volume increase occurred in 10 patients (25\%) with a median time to tumor enlargement of 5.5 months (one-25 months). Prior surgical resection was significantly associated with transient tumor volume increase (Fisher exact, $\mathrm{p}=0.02$ ). Indeed, among the 10 patients with transient tumor volume increase, five patients had prior surgery. The median and mean time-length of transient tumor volume increase were 14 (5-45 months) and 17 months (5-29 months) respectively. Two patients had further surgery after radiotherapy because of increased symptoms or significant brainstem compression. The pathological findings were consistent with residual vestibular schwannoma for both patients without evidence of tumor necrosis.

Prescription dose, GTV volume, PTV volume, maximum dose to PTV, BED, gender, age at treatment and the presence of neurofibromatosis type 2 did not significantly influence the rate of pseudoprogression by Mann-Whitney analysis (Table 2).

TABLE 2: Factors associated with transient tumor volume increase

\section{Cranial nerve toxicities}

Among the 16 (37.2\%) patients who had a serviceable hearing prior to RT, seven patients (47\%) preserved a serviceable hearing and nine patients (53\%) presented with hearing loss (GardnerRobertson grade 3-5) at a mean clinical follow-up of 32 months.

Transient trigeminal neuropathy grade 1-2 according to the CTCAE was observed in four 
patients (9\%) at two years. Patients with prior trigeminal neuropathy did not experience a worsening of their symptoms after RT. No grade 3-4 trigeminal neuropathy was observed.

Among our cohort of patients, three (7\%) developed transient facial neuropathy (grade 2-3) after treatment according to the House-Brackmann score. No grade 4 facial neuropathy was reported. All the patients with radiation-induced cranial nerve toxicities had transient neuropathy, and therefore no medical treatment was needed.

Transient tumor volume increase was statistically associated with an increased rate of facial neuropathy $(\mathrm{p}=0.04)$. There was a trend for increased trigeminal neuropathy with transient tumor volume increase $(\mathrm{p}=0.08$ ) but no association was found with serviceable hearing loss. The prescription dose, homogeneity index, conformity index, isodose line and maximum dose received by the PTV were not correlated with any cranial nerve toxicity.

\section{Discussion}

Discriminating transient tumor volume increase from local failure is difficult but important if one is to avoid unnecessary surgery. In a large series of 452 patients treated over a 10 -year period, 13 patients (3\%) underwent surgery after radiosurgery of whom 10 patients (77\%) had postoperative House Brackmann grade III to VI facial neuropathy [23]. In our study, two patients (4.7\%) had further surgery following radiotherapy.

Transient increase in tumor volume occurred in 10 patients (25\%) of whom five were treated with FSRT (18\%) and five with SRS (33\%). Previous studies reported transient tumor volume increase rates varying between 5-74\% among patients treated with SRS [24-31] and a 48\% rate among patients treated with FSRT [32]. A recent study showed that transient tumor progression after FRST can occur up to three years and is more frequent in larger tumors $(>3 \mathrm{~cm})$ [33]. Some studies found a correlation between tumor enlargement and initial tumor volume after SRS [28], others did not [26,29,31]. In our study, GTV volumes were not associated with transient tumor volume increase $(\mathrm{p}=0.5)$ after SRS or FSRT.

Interestingly, women might have a higher rate of transient tumor volume increase [28]. Seven female patients (32\%) and four male patients (27\%) had transient tumor volume increase $(\mathrm{p}=0.7$ for the correlation of gender and transient tumor increase).

On a Mann Whitney analysis, the prescribed dose, the biological effective dose [17], neurofibromatosis type 2 , and age at treatment were not correlated to transient tumor volume increase. The lack of correlation may be due to the size of our cohort and the heterogeneity of treatments.

In our series, prior surgical resection was significantly associated with transient tumor progression $(\mathrm{p}=0.01)$. This is a new finding. Transient progression may be due to vascular injury. In autopsies of patients dying within weeks of radiation, peri-vascular edema is seen along with thickening of arterial walls. These arterial walls are rich in fibrin, and platelet-fibrin thrombi may be found [34]. These pathologic changes might be exacerbated by previous surgical insult to capillaries and their endothelium.

Another hypothesis as to the pathogenesis of transient post-radiation progression is that radiation injury to tumor cells leads to necrosis and the subsequent release of thrombosis promoting cytokines, cytokines that may also lead to blood-brain barrier disruption. With increased resistance to intravascular outflow, tumors may become congested with blood and increased in size [35]. Indeed, surgical resection of tumors that had tumor volume increase after SRS showed hyalinised thrombosis, thickening of the vascular wall, vascular obstruction, 
and granulomatous changes [24, 36, 37]. Patients that had initial surgery and needed further treatment had either incomplete tumor resection or tumor recurrence. Such "aggressive tumors" with higher cell turnover may suffer greater necrosis and inflammation following radiation.

In our cohort, the median time to transient tumor volume increase after SRS and FSRT was 5.5 months with subsequent tumor shrinkage occurring at a median of 14 months (range: 5-45 months). Similar results have been reported in the literature; the median time to tumor enlargement after SRS varying between six and 13 months [24, 26, 29, 31, 38] with a peak at four to six months $[24,31,39,40]$ followed by tumor shrinkage occurring within two to three years [24, 28, 29]. Therefore, it is reasonable to delay surgery for two years or more [23] in clinically stable and asymptomatic patients.

Little is known about transient tumor volume increase and cranial nerve toxicities. The hearing preservation rate was $47 \%$ at 2 years among patients treated with FSRT and SRS. Hearing preservation rates ranged between 53 and 94\% [19, 41-44] and between 44 and 96\% [43, 45-50] in patients treated with FSRT and SRS, respectively. Although many factors have been analyzed, no statistical association with hearing loss was found. Some studies have associated hearing loss and transient tumor progression [33], other studies did not report a correlation [31]. Predictors of hearing loss after SRS were significant hearing loss before SRS, tumor recurrence and the prescription isodose line, according to a recent study by Wowra et al. [51].

In our study, cranial nerve toxicities rates were low and transient with no permanent facial or trigeminal nerve toxicity. A statistically significant correlation and a trend towards association were found between transient tumor volume increase and increased facial neuropathy $(\mathrm{p}=0.04)$ and trigeminal neuropathy, respectively $(\mathrm{p}=0.08)$. Nagano et al. [31] reported a strong trigeminal and facial nerve dysfunction with transient tumor expansion ( $\mathrm{p}=0.018$ and $\mathrm{p}=0.035$, respectively). On the other hand, Hayhurst et al. [52] did not find an association between tumor pseudoprogression and cranial nerve toxicities. It would seem logical that transient tumor volume increase can cause local compression leading to temporary or permanent cranial neuropathy. Further larger studies are needed to confirm the correlation between transient tumor volume increase and cranial nerve toxicity.

\section{Conclusions}

Transient tumor volume increase is frequent after both SRS and FSRT in VS (25\%). The median time to transient tumor progression was 5.5 months. Transient tumor volume increase was significantly correlated with previous surgery and an increased rate of facial and trigeminal nerve toxicity. The main challenge to the treating physician is to discriminate tumor progression from transient tumor volume increase after radiotherapy in order to avoid unnecessary brain surgery. Conservative management with serial MRIs is recommended for clinically stable patients.

\section{Additional Information}

\section{Disclosures}

Human subjects: Consent was obtained by all participants in this study. The Ethics Committee of the Centre Hospitalier de l'Université de Montréal issued approval \# 10.053. Animal subjects: All authors have confirmed that this study did not involve animal subjects or tissue. Conflicts of interest: In compliance with the ICMJE uniform disclosure form, all authors declare the following: Payment/services info: All authors have declared that no financial support was received from any organization for the submitted work. Financial relationships: All authors have declared that they have no financial relationships at present or within the 
previous three years with any organizations that might have an interest in the submitted work. Other relationships: All authors have declared that there are no other relationships or activities that could appear to have influenced the submitted work.

\section{Acknowledgements}

We would like to thank Dr. Daniel Taussky for his collaboration and for his support in this study.

\section{References}

1. Mirz, F., Jorgensen, B., Fiirgaard, B., Lundorf, E., Pedersen, C. B. : Investigations into the natural history of vestibular schwannomas. Clin Otolaryngol Allied Sci. 1999, 24:13-18.

2. Yamakami, I., Uchino, Y., Kobayashi, E., Yamaura, A: Conservative management, gammaknife radiosurgery, and microsurgery for acoustic neurinomas: a systematic review of outcome and risk of three therapeutic options. Neurological Research. 2003, 25:682-690.

3. Kaylie, D. M., Horgan, M. J., Delashaw, J. B., McMenomey, S. O: A meta-analysis comparing outcomes of microsurgery and gamma knife radiosurgery. The Laryngoscope. 2000, 110:18501856.

4. Gormley, W. B., Sekhar, L. N., Wright, D. C., Kamerer, D., Schessel, D: Acoustic neuromas: results of current surgical management. Neurosurgery. 1997, 41:50-58.

5. Samii, M., Matthies, C: Management of 1000 vestibular schwannomas (acoustic neuromas): The facial nerve--preservation and restitution of function. Neurosurgery. 1997, 40:684-694.

6. Falcioni, M., Mulder, J. J., Taibah, A., De Donato, G., Sanna, M: No cerebrospinal fluid leaks in translabyrinthine vestibular schwannoma removal: Reappraisal of 200 consecutive patients. Am J Otol. 1999, 20:660-666.

7. Darrouzet, V., Martel, J., Enee, V., Bebear, J. P., Guerin, J: Vestibular schwannoma surgery outcomes: our multidisciplinary experience in 400 cases over 17 years. The Laryngoscope. 2004, 114:681-688.

8. Pollock, B. E., Driscoll, C. L., Foote, R. L., Link, M. J., Gorman, D. A., Bauch, C. D., Mandrekar, J. N., Krecke, K. N., Johnson, C. H: Patient outcomes after vestibular schwannoma management: a prospective comparison of microsurgical resection and stereotactic radiosurgery. Neurosurgery. 2006, 59:77-85.

9. Samii, M., Gerganov, V., Samii, A: Improved preservation of hearing and facial nerve function in vestibular schwannoma surgery via the retrosigmoid approach in a series of 200 patients. Journal of Neurosurgery. 2006, 105:527-535.

10. Arthurs, B. J., Fairbanks, R. K., Demakas, J. J., Lamoreaux, W. T., Giddings, N. A., Mackay, A. R., Cooke, B. S., Elaimy, A. L., Lee, C. M: A review of treatment modalities for vestibular schwannoma. Neurosurg Rev. 2011, 34:265-277.

11. Yoshimoto, Y: Systematic review of the natural history of vestibular schwannoma . Journal of Neurosurgery. 2005, 103:59-63.

12. Sughrue, M. E., Yang, I., Aranda, D., Lobo, K., Pitts, L. H., Cheung, S. W., Parsa, A. T: The natural history of untreated sporadic vestibular schwannomas: a comprehensive review of hearing outcomes. Journal of Neurosurgery. 2010, 112:163-167.

13. Battaglia, A., Mastrodimos, B., Cueva, R: Comparison of growth patterns of acoustic neuromas with and without radiosurgery. Otol Neurotol. 2006, 27:705-712.

14. Selesnick, S. H., Johnson, G: Radiologic surveillance of acoustic neuromas. Am J Otol. 1998, 19:846-849.

15. Smouha, E. E., Yoo, M., Mohr, K., Davis, R. P: Conservative management of acoustic neuroma: a meta-analysis and proposed treatment algorithm. The Laryngoscope. 2005, 115:450-454.

16. Plotkin, S. R., Stemmer-Rachamimov, A. O., Barker, F. G., 2nd, Halpin, C., Padera, T. P., Tyrrell, A., Sorensen, A. G., Jain, R. K., di Tomaso, E: Hearing improvement after bevacizumab in patients with neurofibromatosis type 2. NEJM. 2009, 361:358-367.

17. Andrews, D. W., Werner-Wasik, M., Den, R. B., Paek, S. H., Downes-Phillips, B., Willcox, T. O., Bednarz, G., Maltenfort, M., Evans, J. J., Curran, W. J., Jr: Toward dose optimization for fractionated stereotactic radiotherapy for acoustic neuromas: comparison of two dose cohorts. Int J Radiat Oncol Biol Phys. 2009, 74:419-426. 
18. Chan, A. W., Black, P., Ojemann, R. G., Barker, F. G., 2nd, Kooy, H. M., Lopes, V. V., McKenna, M. J., Shrieve, D. C., Martuza, R. L., Loeffler, J. S: Stereotactic radiotherapy for vestibular schwannomas: Favorable outcome with minimal toxicity. Neurosurgery. 2005, 57:60-70.

19. Combs, S. E., Volk, S., Schulz-Ertner, D., Huber, P. E., Thilmann, C., Debus, J: Management of acoustic neuromas with fractionated stereotactic radiotherapy (FSRT): long-term results in 106 patients treated in a single institution. Int J Radiat Oncol Biol Phys. 2005, 63:75-81.

20. Koh, E. S., Millar, B. A., Menard, C., Michaels, H., Heydarian, M., Ladak, S., McKinnon, S., Rutka, J. A., Guha, A., Pond, G. R., Laperriere, N. J: Fractionated stereotactic radiotherapy for acoustic neuroma: single-institution experience at The Princess Margaret Hospital. Cancer. 2007, 109:1203-1210.

21. Thomas, C., Di Maio, S., Ma, R., Vollans, E., Chu, C., Clark, B., Lee, R., McKenzie, M., Martin, M., Toyota, B: Hearing preservation following fractionated stereotactic radiotherapy for vestibular schwannomas: prognostic implications of cochlear dose. Journal of Neurosurgery. 2007, 107:917-926.

22. Flickinger, J. C., Kondziolka, D., Niranjan, A., Maitz, A., Voynov, G., Lunsford, L. D: Acoustic neuroma radiosurgery with marginal tumor doses of 12 to $13 \mathrm{~Gy}$. Int J Radiat Oncol Biol Phys. 2004, 60:225-230.

23. Pollock, B. E., Lunsford, L. D., Kondziolka, D., Sekula, R., Subach, B. R., Foote, R. L., Flickinger, J. C: Vestibular schwannoma management. Part II. Failed radiosurgery and the role of delayed microsurgery. Journal of Neurosurgery. 1998, 89:949-955.

24. Kim, K. M., Park, C. K., Chung, H. T., Paek, S. H., Jung, H. W., Kim, D. G: Long-term Outcomes of Gamma Knife Stereotactic Radiosurgery of Vestibular Schwannomas. J Korean Neurosurg Soc. 2007, 42:286-292.

25. Noren, G: Long-term complications following gamma knife radiosurgery of vestibular schwannomas. Stereotact Funct Neurosurg. 1998, 70 :65-73.

26. Pollock, B. E: Management of vestibular schwannomas that enlarge after stereotactic radiosurgery: treatment recommendations based on a 15 year experience. Neurosurgery. 2006, 58:241-248.

27. Delsanti, C., Tamura, M., Galanaud, D., Regis J: Changing radiological results, pitfalls and criteria of failure. Neurochirurgie. 2004, 50:312-319.

28. Hasegawa, T., Kida, Y., Yoshimoto, M., Koike, J., Goto, K: Evaluation of tumor expansion after stereotactic radiosurgery in patients harboring vestibular schwannomas. Neurosurgery. 2006, 58:1119-1128.

29. Nakamura, H., Jokura, H., Takahashi, K., Boku, N., Akabane, A., Yoshimoto, T: Serial followup MR imaging after gamma knife radiosurgery for vestibular schwannoma. AJNR. 2000, 21:1540-1546.

30. Yu, C. P., Cheung, J. Y., Leung, S., Ho, R: Sequential volume mapping for confirmation of negative growth in vestibular schwannomas treated by gamma knife radiosurgery. Journal of Neurosurgery. 2000, 93:82-89.

31. Nagano, O., Higuchi, Y., Serizawa, T., Ono, J., Matsuda, S., Yamakami, I., Saeki, N: Transient expansion of vestibular schwannoma following stereotactic radiosurgery. Journal of Neurosurgery. 2008, 109:811-816.

32. Sakanaka, K., Mizowaki, T., Arakawa, Y., Araki, N., Oya, N., Takahashi, J. A., Mikuni, N., Miyamoto, S., Hashimoto, N., Hiraoka, M: Hypofractionated stereotactic radiotherapy for acoustic neuromas: safety and effectiveness over 8 years of experience. Int J Clin Oncol. 2011, 16:27-32.

33. Aoyama, H., Takeichi, N., Onodera, S., Taguchi, H., Sawamura, Y., Shirato, H: Conventionally Fractionated Stereotactic Radiotherapy for Vestibular Schwannoma: A Single Institutional Long-term Outcomes. Int J Radiat Oncol Biol Phys. 2010, 78:S8-S9.

34. Fajardo, L. F., Berthrong, M., Anderson, R. E: Radiation Pathology. Oxford University Press, New York, NY; 2001.

35. Suzuki, H., Toyoda, S., Muramatsu, M., Shimizu, T., Kojima, T., Taki, W: Spontaneous haemorrhage into metastatic brain tumours after stereotactic radiosurgery using a linear accelerator. J Neurol Neurosurg Psychiatry. 2003, 74:908-912.

36. Hirato, M., Inoue, H., Nakamura, M., Ohye, C., Hirato, J., Shibazaki, T., Andou, Y: Gamma Knife radiosurgery for acoustic schwannoma: Early effects and preservation of hearing. Neurol Med Chir (Tokyo). 1995, 35,:737-741.

37. Kobayashi, T., Tanaka, T., Kida, Y: The early effects of gamma knife on 40 cases of acoustic 
neurinoma. Acta Neurochir Suppl. 1994, 62:93-97.

38. Oyama, H., Kobayashi, T., Kida, Y., Tanaka, T., Mori, Y., Iwakoshi, T., Niwa, M., Kai, O., Hirose, M: Early changes in volume and non-enhanced volume of acoustic neurinoma after stereotactic gamma-radiosurgery. Neurol Med Chir (Tokyo). 1994, 34:607-611.

39. Okunaga, T., Matsuo, T., Hayashi, N., Hayashi, Y., Shabani, H. K., Kaminogo, M., Ochi, M., Nagata, I: Linear accelerator radiosurgery for vestibular schwannoma: Measuring tumor volume changes on serial three-dimensional spoiled gradient-echo magnetic resonance images. Journal of Neurosurgery. 2005, 103:53-58.

40. Carai, A., Green, S., Delman, B., Blacksburg, S. R., Maloney-Lutz, K., Lo, Y., Sheu, R., Germano, I: Tumor Volume Increase after Stereotactic Radiosurgery for Vestibular Schwannoma: Expected Radiographic Finding or Exception?. Int J Radiat Oncol Biol Phys. 2010, 78:S280.

41. Meijer, O. W., Vandertop, W. P., Baayen, J. C., Slotman, B. J: Single-fraction vs. fractionated linac-based stereotactic radiosurgery for vestibular schwannoma: a single-institution study. Int J Radiat Oncol Biol Phys. 2003, 56:1390-1396.

42. Sawamura, Y., Shirato, H., Sakamoto, T., Aoyama, H., Suzuki, K., Onimaru, R., Isu, T., Fukuda, S., Miyasaka, K: Management of vestibular schwannoma by fractionated stereotactic radiotherapy and associated cerebrospinal fluid malabsorption. Journal of Neurosurgery. 2003, 99:685-692.

43. Combs, S. E., Welzel, T., Schulz-Ertner, D., Huber, P. E., Debus, J: Differences in clinical results after LINAC-based single-dose radiosurgery versus fractionated stereotactic radiotherapy for patients with vestibular schwannomas. Int J Radiat Oncol Biol Phys. 2010, 76:193-200.

44. Shirato, H., Sakamoto, T., Sawamura, Y., Kagei, K., Isu, T., Kato, T., Fukuda, S., Suzuki, K., Soma, S., Inuyama, Y., Miyasaka, K: Comparison between observation policy and fractionated stereotactic radiotherapy (SRT) as an initial management for vestibular schwannoma. Int J Radiat Oncol Biol Phys. 1999, 44:545-550.

45. Spiegelmann, R., Gofman, J., Alezra, D., Pfeffer, R: Radiosurgery for acoustic neurinomas vestibular schwannomas. IMAJ. 1999, 1:8-13.

46. Spiegelmann, R., Lidar, Z., Gofman, J., Alezra, D., Hadani, M., Pfeffer, R: Linear accelerator radiosurgery for vestibular schwannoma. Journal of Neurosurgery. 2001, 94:7-13.

47. Chang, S. D., Gibbs, I. C., Sakamoto, G. T., Lee, E., Oyelese, A., Adler, J. R., Jr: Staged stereotactic irradiation for acoustic neuroma. Neurosurgery. 2005, 56:1254-1261.

48. Rutten, I., Baumert, B. G., Seidel, L., Kotolenko, S., Collignon, J., Kaschten, B., Albert, A., Martin, D., Deneufbourg, J. M., Demanez, J. P., Stevenaert: A. Long-term follow-up reveals low toxicity of radiosurgery for vestibular schwannoma. Radiother Oncol. 2007, 82:83-89.

49. Chopra, R., Kondziolka, D., Niranjan, A., Lunsford, L. D., Flickinger, J. C: Long-term follow-up of acoustic schwannoma radiosurgery with marginal tumor doses of 12 to $13 \mathrm{~Gy}$. Int J Radiat Oncol Biol Phys. 2007, 68:845-851.

50. Combs, S. E., Thilmann, C., Debus, J., Schulz-Ertner, D: Long-term outcome of stereotactic radiosurgery (SRS) in patients with acoustic neuromas. Int J Radiat Oncol Biol Phys. 2006, 64:1341-1347.

51. Wowra, B., Muacevic, A., Furweger, C., Schichor, C., Tonn, J. C: Therapeutic profile of singlefraction radiosurgery of vestibular schwannoma: Unrelated malignancy predicts tumor control. Neuro-oncology. 2012, 14:902-909.

52. Hayhurst, C., Zadeh, G: Tumor pseudoprogression following radiosurgery for vestibular schwannoma. Neuro-oncology. 2012, 14:87-92. 